\title{
The characteristic of unsaturated polyester resin wettability toward glass fiber orientation, density and surface treatment
}

\author{
Asep H. Saputra ${ }^{1, *}$ and Dena P. Hallatu ${ }^{1}$ \\ ${ }^{1}$ Department of Chemical Engineering, Faculty of Engineering, Universitas Indonesia, Depok 16424, Indonesia
}

\begin{abstract}
Wettability of composite is one of key to increase mechanical properties of composite that affected by structure of reinforcement and type of resin used. Therefore, this research focused on the effect of orientation, density and surface treatment on fiber to the characteristic of composite's wettability, which is observed by contact angle and wetting time. The fiber used in this research is fiberglass, and the method for contact angle measurement is direct observation from the camera recorder and the data record will be processed and analyzed by using image processing method. The result for those variations can be obtained from the relation of variations toward contact angle and wetting time. According to result of research, fiber with orientation $45^{\circ} / 45^{\circ}$ gives lower contact angle but longer wetting time than fiber with orientation $0^{\circ} / 90^{\circ}$. For orientation $45^{\circ} / 45^{\circ}$, the differences in wetting time is 15 second longer than orientation $0^{\circ} / 90^{\circ}$. In case of fiber density, the sheet with fiber density of 900 has 7 second faster for wetting time than sheet with fiber density of 1250 . The surface treatment with $\mathrm{NaOH} 5 \%$ can accelerate the wetting time until 10 second.
\end{abstract}

\section{Introduction}

Composite material is often preferred because it has good mechanical properties. The type of matrix, the reinforcement used, and the interaction between matrix and reinforcement can affect mechanical properties of composite material. Wettability is the ability of liquid 'wetting' the surface of a solid material, as in how composite matrix wet reinforcement particles. Optimizing the ability of a matrix to wet the reinforcement is a key for increasing the mechanical properties of composite material.

Wettability properties of a material depend on hydrophobic properties and viscosity of liquid. Viscosity affect hydrophobic properties by increasing the contact angle through liquid viscosity decrease, and the rough surface effect might not always create high hydrophobic properties [9]. Reinforcement materials have a portion in surface free energy used for composite material wettability measurement [3].

Contact angle measurement is one of the method that is used to observe and develop the wetting phenomena. This method is commonly used because the measurement characteristic does not damage experiment materials nor alter the chemical and physical properties of material [2]. Contact angle is obtained by measuring the angle between material surface and liquid in which both has different polarity. Contact angle and wettability both depend on the time of substrate drop and the time of image data taken to measure the contact angle. [4].
Several methods are commonly used in the measurement of contact angle. One of the methods is by measuring the angle between composite material surface and the liquid drop. In accordance to the development of technology in computation, tools for contact angle measurement has been developed with better accuracy and precision. The uses of camera which providing high zoom capability as a tool to capture liquid drop profile provide the measurement of profile on detail [8]. Image processing method for contact angle measurement has the potential to measure the contact angle from any type and shape of liquid drop [6]. Identification with image processing method gives several advantages, such as increasing the sensitivity of experiment and resulting in better accuracy within the number of image that can be analyzed by computer [1].

Since wettability of composite is one of key factor in composite fabrication which affected by the reinforcement and resin, the effect of fiber orientation and fiber sheet density, and fiber surface treatment to composite wettability is needed to be observed.

\section{Experimental details}

\subsection{Materials and tools}

Materials used for research are fiberglass and unsaturated polyester resin. Unsaturated polyester resin which is used in this research is EVERPOL 324 AR-2

\footnotetext{
* Corresponding author: author@e-mail.org
} 
supplied by PT. Arindo Pacific Chemicals. Resin type is ortho with clear pale color and has 560 cps of viscosity

The type of fiberglass used has a varied orientation and sheet density. Type of fiberglass used are Biax Combi $900\left(0^{\circ} / 90^{\circ} / \mathrm{M} 300\right.$, Width $\left.127 \mathrm{~cm}\right)$, Biax Combi $1250\left(0^{\circ} / 90^{\circ} / \mathrm{M} 450\right.$, Width $\left.127 \mathrm{~cm}\right)$, Biax Combi 900 $\left(+45^{\circ} / 45^{\circ} / \mathrm{M} 300\right.$, Width $127 \mathrm{~cm}$, and Biax Combi 1250 $\left(+45^{\circ} / 45^{\circ} / \mathrm{M} 450\right.$, Width $\left.127 \mathrm{~cm}\right)$. The number of 900 and 1250 indicate the density of fiber, while $0 \% / 90^{\circ}$ and $+45^{\circ} / 45$ indicate fiber orientation.

The tools used in this research are camera with tripod and dropper/pipette. Using tripod gives optimal results, because it holds camera on constant position along the research. Camera that used has capacity 60 frame per second and standard lens with $55 \mathrm{~mm}$ zoom.

\subsection{Data collection process}

The dependent variables in this research that will be observed are contact angle and wetting time. The method used for contact angle measurement is image processing method by using the tools as seen in Figure 1.

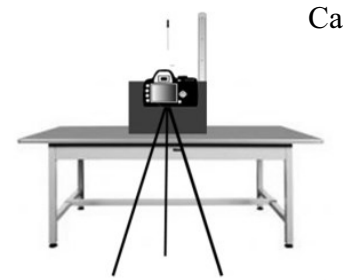

(a)

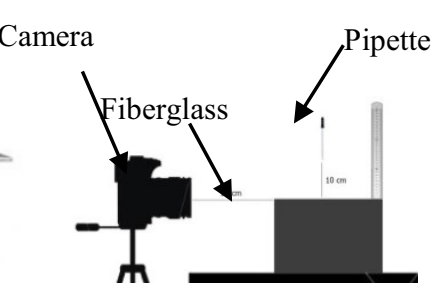

(b)
Fig. 1. Scheme of Experimental Tools; (a) front view; (b) side view

The use of sections to divide the text of the paper is optional and left as a decision for the author. Where the author wishes to divide the paper into sections the formatting shown in Table 2 should be used. The data collection process in this research is contact angle in degree $\left({ }^{\circ}\right)$ and wetting time in second (s). The form of liquid droplet dropped from pipette to fiber surface is assumed to be in the form of sessile drop. The data is taken with a camera to record all of the process beginning with the time of resin dropping up until the resin completely disappeared from fiber surface. To provide data with higher precision, the camera is supported with tripod. Considering that this experiment depends on tools positioning, all of the tools used such as camera, pipette and experimental table are placed in same position during the observation.

The experiment material, fiberglass, is placed in a table and pipette, which is a tool used for dropping the liquid is placed $8 \mathrm{~cm}$ above the table. Camera is set in line with experiment table and $25 \mathrm{~cm}$ from it. Resin dropped from pipette to fiberglass surface and recorded with camera. The camera is in recorder mode. The video results will be converted to image by using software converter for 60 images (frames) in 1 second. Data for contact angle is taken in frame $1(0 \mathrm{~s}), 50(0.83 \mathrm{~s})$ and $100(1.67 \mathrm{~s})$. In that frame, contact angle will be measured using software. Meanwhile, wetting time is obtained from data recorder. Wetting time is condition when liquid droplet completely disappeared from fiberglass surface or it can be defined as condition when contact angle reaches 0 degree.

\section{Results and discussion}

This research is focused on the effect of fiber orientation, fiber density and surface treatment with alkali solution ( $\mathrm{NaOH} \mathrm{5 \% )} \mathrm{which} \mathrm{is} \mathrm{observed} \mathrm{through} \mathrm{contact} \mathrm{angle} \mathrm{and}$ wetting time, since wetting time is one of important thing in manufacturing process of composite materials, especially in large object with many layers.

\subsection{The effect of fiber orientation in fiberglass without surface treatment}

The effect of fiber orientation to the contact angle and wetting time is observed. Fiber orientation which is variation in this research are fiber with orientation $0^{\circ} / 90^{\circ}$ and fiber with orientation $45^{\circ} / 45^{\circ}$. The data was obtained from the condition in which fiber has same fiber density. This data will be compared between the sheet with fiber density of 900 and the sheet with fiber density of 1250 .
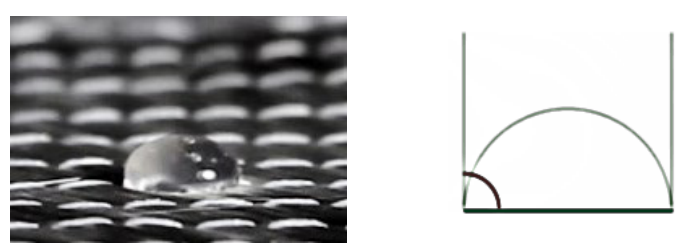

(a)
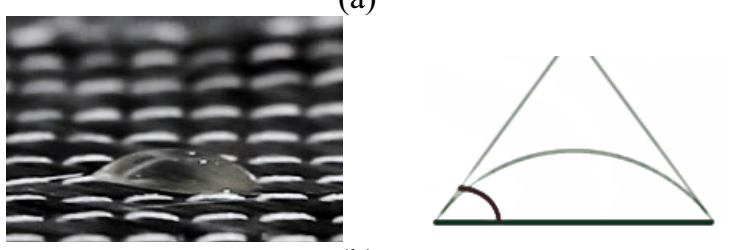

(b)
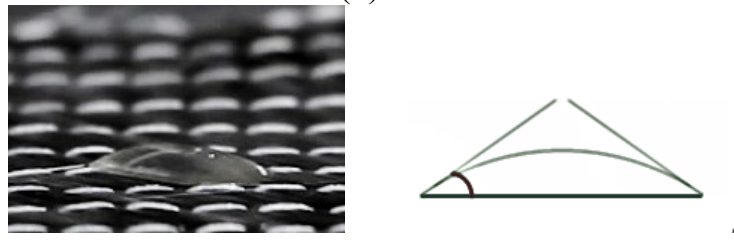

(c)

Fig. 2. Experimental Results Example for Fiber without Surface Treatment; (a) Frame 1 (0 s); (b) Frame 50 (0.83 s); (c) Frame 100 (1.67 s)

Figure 2 shows the typically form of liquid droplet in fiberglass surface. Contact angle data is get from measurement angle between fiberglass surface and liquid droplet, as seen as Figure 2 illustration. As seen in figure since the increasing of time, contact angle will be decrease and disappear from fiberglass surface. 
Table 1. Contact Angle of the Sheet with Fiber Density of 900 for the Fiber without Surface Treatment.

\begin{tabular}{|c|c|c|c|}
\hline \multirow{2}{*}{ Orientation } & \multicolumn{3}{|c|}{ Contact Angle } \\
\cline { 2 - 4 } & $\begin{array}{c}\text { Frame 1 } \\
\mathbf{( 0 ~ s )}\end{array}$ & $\begin{array}{c}\text { Frame 50 } \\
\mathbf{( 0 . 8 3 ~ s )}\end{array}$ & $\begin{array}{c}\text { Frame 100 } \\
\mathbf{( 1 . 6 7 ~ s )}\end{array}$ \\
\hline $0^{\circ} / 90^{\circ}$ & $\theta=88.752$ & $\theta=49.223$ & $\theta=37.168$ \\
\hline $45^{\circ} / 45^{\circ}$ & $\theta=88.707$ & $\theta=49.144$ & $\theta=36.044$ \\
\hline
\end{tabular}

Table 2. Contact Angle of the Sheet with Fiber Density of 1250 for the Fiber without Surface Treatment

\begin{tabular}{|c|c|c|c|}
\hline \multirow{2}{*}{ Orientation } & \multicolumn{3}{|c|}{ Contact Angle } \\
\cline { 2 - 4 } & $\begin{array}{c}\text { Frame 1 } \\
\mathbf{( 0 ~ s )}\end{array}$ & $\begin{array}{c}\text { Frame 50 } \\
\mathbf{( 0 . 8 3 ~ s )}\end{array}$ & $\begin{array}{c}\text { Frame 100 } \\
(\mathbf{1 . 6 7} \mathbf{~ s})\end{array}$ \\
\hline $0^{\circ} / 90^{\circ}$ & $\theta=91.586$ & $\theta=61.994$ & $\theta=45.354$ \\
\hline $45^{\circ} / 45^{\circ}$ & $\theta=90.355$ & $\theta=52.277$ & $\theta=39.073$ \\
\hline
\end{tabular}

The results for contact angle measurement for sheet with fiber density of 900 and sheet with fiber density of 1250 is given in Table 1 and Table 2. Both of sheet with fiber density of 900 and sheet with fiber density of 1250 has results with the same tendency. Contact angle result from fiber with orientation $0^{\circ} / 90$ is higher than result from fiber with orientation $45^{\circ} / 45^{\circ}$. The difference reaches 6 degree in frame 100 . Besides from the contact angle, wettability is also can be observed from the wetting time.

Table 3. Wetting Time of Fiber without Surface Treatment

\begin{tabular}{|c|c|c|}
\hline Fiber Density & Fiber Orientation & Wetting Time (s) \\
\hline \multirow{2}{*}{900} & $0^{\circ} / 90^{\circ}$ & 32.00 \\
\cline { 2 - 3 } & $45^{\circ} / 45^{\circ}$ & 45.33 \\
\hline \multirow{2}{*}{1250} & $0^{\circ} / 90^{\circ}$ & 35.00 \\
\cline { 2 - 3 } & $45^{\circ} / 45^{\circ}$ & 48.67 \\
\hline
\end{tabular}

Table 3 shows that the fiber with orientation $0^{\circ} / 90^{\circ}$ has faster wetting time than the fiber with orientation $45^{\circ} / 45^{\circ}$. This result happens in either sheet with fiber density of 900 or sheet with fiber density of 1250 . Fiber with orientation $0^{\circ} / 90^{\circ}$ has 13 second faster in wetting time than fiber with orientation $45^{\circ} / 45^{\circ}$.

The result for contact angle and wetting time measurement shows that the fiber with orientation $0^{\circ} / 90^{\circ}$ has higher contact angle and faster wetting time than fiber with orientation $45 \%$. It happens because in contact angle measurement, the droplet formed depends on early surface contact with the droplet while wetting time depends on fiber space areas in fiber sheet surface. The fiber with orientation $0^{\circ} / 90^{\circ}$ has wider area than fiber with orientation $45^{\circ} / 45^{\circ}$ that makes the wetting time faster.

\subsection{The effect of fiber density in fiberglass without surface treatment}

The effect of fiber density to the contact angle and wetting time is observed. Density which will be compared in research is sheet with fiber density of 900 and sheet with fiber density of 1250 .

Table 4. Contact Angle of Fiber with Orientation $0^{\circ} / 90^{\circ}$ in Fiber without Surface Treatment

\begin{tabular}{|c|c|c|c|}
\hline \multirow{2}{*}{ Density } & \multicolumn{3}{|c|}{ Contact Angle } \\
\cline { 2 - 4 } & $\begin{array}{c}\text { Frame 1 } \\
\mathbf{( 0 ~ s )}\end{array}$ & $\begin{array}{c}\text { Frame 50 } \\
\mathbf{( 0 . 8 3} \mathbf{~ s})\end{array}$ & $\begin{array}{c}\text { Frame 100 } \\
\mathbf{( 1 . 6 7} \mathbf{~ s})\end{array}$ \\
\hline 900 & $\theta=88.752$ & $\theta=49.223$ & $\theta=37.168$ \\
\hline 1250 & $\theta=91.586$ & $\theta=61.994$ & $\theta=45.354$ \\
\hline
\end{tabular}

Table 5. Contact Angle of Fiber with Orientation $45^{\circ} / 45^{\circ}$ in Fiber without Surface Treatment

\begin{tabular}{|c|c|c|c|}
\hline \multirow{2}{*}{ Density } & \multicolumn{3}{|c|}{ Contact Angle } \\
\cline { 2 - 4 } & $\begin{array}{c}\text { Frame 1 } \\
\mathbf{( 0 ~ s )}\end{array}$ & $\begin{array}{c}\text { Frame 50 } \\
\mathbf{( 0 . 8 3} \mathbf{~ s )}\end{array}$ & $\begin{array}{c}\text { Frame 100 } \\
\mathbf{( 1 . 6 7} \mathbf{~ s})\end{array}$ \\
\hline 900 & $\theta=88.707$ & $\theta=49.144$ & $\theta=36.044$ \\
\hline 1250 & $\theta=90.355$ & $\theta=52.277$ & $\theta=39.073$ \\
\hline
\end{tabular}

Table 4 and Table 5 shows the effect of fiber density to the contact angle. Based on the results, both of fiber with orientation $0^{\circ} / 90^{\circ}$ or fiber with orientation $45^{\circ} / 45^{\circ}$ shows that contact angle in the sheet with fiber density of 900 is lower than the sheet with fiber density of 1250 . In addition, wettability can also be observed from the wetting time.

Table 6. Wetting Time of Fiber without Surface Treatment

\begin{tabular}{|c|c|c|}
\hline Fiber Density & Fiber Orientation & Wetting Time (s) \\
\hline \multirow{2}{*}{900} & $0 \% / 90^{\circ}$ & 32.00 \\
\cline { 2 - 3 } & $45^{\circ} / 45^{\circ}$ & 45.33 \\
\hline \multirow{2}{*}{1250} & $0^{\circ} / 90^{\circ}$ & 35.00 \\
\cline { 2 - 3 } & $45^{\circ} / 45^{\circ}$ & 48.67 \\
\hline
\end{tabular}

As seen also in Table 6, the results shows that sheet with fiber density of 900 has lower contact angle and faster wetting time than sheet with fiber density of 1250 . Sheet 
with fiber density of 900 has 3 second faster than sheet with fiber density of 1250 . When the density of fiber is tighter, it will be more difficult for liquid to enter the fiber surface because the space area provided in the fiber surface is too small for the liquid droplet to enter.

\subsection{The effect of fiber orientation in fiberglass with surface treatment ( $\mathrm{NaOH} 5 \%$ )}

The surface treatment in this research is performed by submerging the fiberglass with alkali ( $\mathrm{NaOH} 5 \%$ ) solution. The purpose of the submersion is to discover the effect of surface treatment on fiber to the contact angle and wetting time.
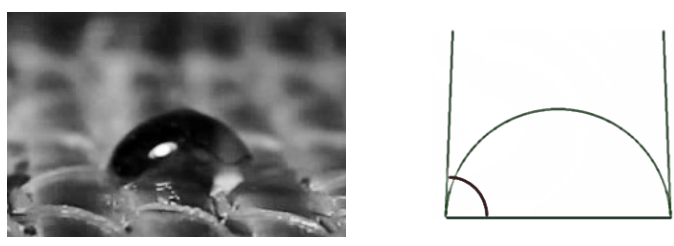

(a)
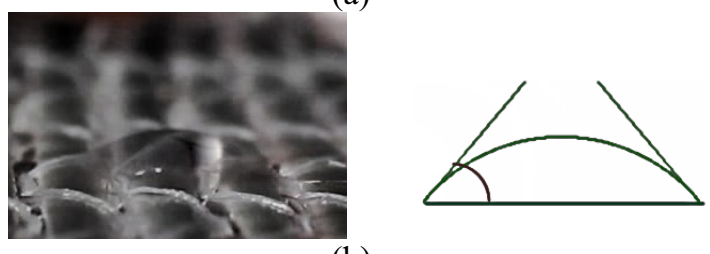

(b)
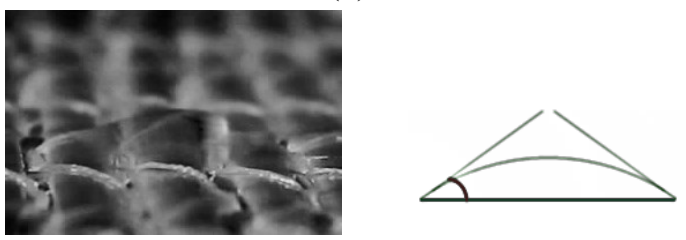

(b)

Fig. 3. Experimental Results Example for Fiber with Surface Treatment; (a) Frame 1 (0 s); (b) Frame 50 (0.83 s); (c) Frame $100(1.67 \mathrm{~s})$

Figure 3 shows the example of image taken in research for fiberglass with surface treatment. Image data will be using in contact angle measurement with software analysis and give the exact contact angle. Contact angle data is get from measurement angle between fiberglass surface and liquid droplet, as seen as Figure 3 illustration. Since the increasing of time, contact angle will be decrease and disappear from fiberglass surface. The data was obtained from the condition in which fiber has same density. This data is then compared between the sheet with fiber density of 900 and the sheet with fiber density of 1250 . The results for contact angle measurement for sheet with fiber density of 900 and sheet with fiber density of 1250 will be given in Table 7 and Table 8 . Table 7 and Table 8 shows that both of the sheet has the same tendency. Contact angle resulted from fiber with orientation $0 \%$ is higher than the one resulted from fiber with orientation $45^{\circ} / 45^{\circ}$. Table 9 showed that fiber with orientation $0^{\circ} / 90^{\circ}$ need faster time than fiber with orientation $45^{\circ} / 45$, it happens in either sheet with fiber density of 900 or sheet with fiber density of 1250 . Furthermore, wettability can also be observed from the wetting time.

Table 7. Contact Angle of Sheet with Fiber Density of 900 in Fiber with Surface Treatment

\begin{tabular}{|c|c|c|c|}
\hline \multirow{2}{*}{ Orientation } & \multicolumn{3}{|c|}{ Contact Angle } \\
\cline { 2 - 4 } & $\begin{array}{c}\text { Frame 1 } \\
\mathbf{( 0 ~ s )}\end{array}$ & $\begin{array}{c}\text { Frame 50 } \\
\mathbf{( 0 . 8 3} \mathbf{~ s )}\end{array}$ & $\begin{array}{c}\text { Frame 100 } \\
\mathbf{( 1 . 6 7 ~ s )}\end{array}$ \\
\hline $0^{\circ} / 90^{\circ}$ & $\theta=87.698$ & $\theta=43.029$ & $\theta=34.397$ \\
\hline $45^{\circ} / 45^{\circ}$ & $\theta=87.498$ & $\theta=42.000$ & $\theta=32.294$ \\
\hline
\end{tabular}

Table 8. Contact Angle of Sheet with Fiber Density of 1250 in Fiber with Surface Treatment

\begin{tabular}{|c|c|c|c|}
\hline \multirow{2}{*}{ Orientation } & \multicolumn{3}{|c|}{ Contact Angle } \\
\cline { 2 - 4 } & $\begin{array}{c}\text { Frame 1 } \\
\mathbf{( 0 ~ s )}\end{array}$ & $\begin{array}{c}\text { Frame 50 } \\
\mathbf{( 0 . 8 3} \mathbf{~ s})\end{array}$ & $\begin{array}{c}\text { Frame 100 } \\
\mathbf{( 1 . 6 7 ~ s )}\end{array}$ \\
\hline $0^{\circ} / 90^{\circ}$ & $\theta=88.727$ & $\theta=47.373$ & $\theta=40.997$ \\
\hline $45^{\circ} / 45^{\circ}$ & $\theta=88.031$ & $\theta=46.483$ & $\theta=35.066$ \\
\hline
\end{tabular}

Table 9. Wetting Time for Fiber with Surface Treatment

\begin{tabular}{|c|c|c|}
\hline Fiber Density & Fiber Orientation & Wetting Time (s) \\
\hline \multirow{2}{*}{900} & $0^{\circ} / 90^{\circ}$ & 19.00 \\
\cline { 2 - 3 } & $45^{\circ} / 45^{\circ}$ & 36.33 \\
\hline \multirow{2}{*}{1250} & $0^{\circ} / 90^{\circ}$ & 19.67 \\
\cline { 2 - 3 } & $45^{\circ} / 45^{\circ}$ & 37.00 \\
\hline
\end{tabular}

In addition, fiber with orientation $0^{\circ} / 90^{\circ}$ has higher contact angle and faster wetting time than fiber with orientation $45 \% 45$. It happens because the contact angle measurement depends on the early surface contact with the droplet, while the wetting time depends on fiber space areas in surface of fiber sheet. The fiber with orientation $0^{\circ} / 90^{\circ}$ has wider area than fiber with orientation $45^{\circ} / 45^{\circ}$ which makes the wetting become faster. Both of using surface treatment or without surface treatment, the results give the same tendency, even though fiber with surface treatment gives lower contact angle and faster wetting time.

\subsection{The effect of fiber orientation in fiberglass with surface treatment ( $\mathrm{NaOH} 5 \%)$}

The effect of fiber density to the contact angle and wetting time is observed. The data was obtained from the condition in which fiber has same fiber orientation. This 
data is then compared between fiber with orientation $0^{\circ} / 90^{\circ}$ and fiber with orientation $45^{\circ} / 45^{\circ}$.

Table 10. Contact Angle of Fiber with Orientation $0^{\circ} / 90^{\circ}$ in Fiber with Surface Treatment

\begin{tabular}{|c|c|c|c|}
\hline \multirow{2}{*}{ Density } & \multicolumn{3}{|c|}{ Contact Angle } \\
\cline { 2 - 4 } & $\begin{array}{c}\text { Frame 1 } \\
\mathbf{( 0 ~ s )}\end{array}$ & $\begin{array}{c}\text { Frame 50 } \\
\mathbf{( 0 . 8 3} \text { s) }\end{array}$ & $\begin{array}{c}\text { Frame 100 } \\
(\mathbf{1 . 6 7} \text { s) }\end{array}$ \\
\hline 900 & $\theta=87.698$ & $\theta=43.029$ & $\theta=34.397$ \\
\hline 1250 & $\theta=88.727$ & $\theta=47.373$ & $\theta=40.997$ \\
\hline
\end{tabular}

Table 11. Contact Angle of Fiber with Orientation $0^{\circ} / 90^{\circ}$ in Fiber with Surface Treatment

\begin{tabular}{|c|c|c|c|}
\hline \multirow{2}{*}{ Density } & \multicolumn{3}{|c|}{ Contact Angle } \\
\cline { 2 - 4 } & $\begin{array}{c}\text { Frame 1 } \\
(\mathbf{0} \text { s) }\end{array}$ & $\begin{array}{c}\text { Frame 50 } \\
\mathbf{( 0 . 8 3} \mathbf{~ s})\end{array}$ & $\begin{array}{c}\text { Frame 100 } \\
\mathbf{( 1 . 6 7} \mathbf{~ s})\end{array}$ \\
\hline 900 & $\theta=87.498$ & $\theta=42.000$ & $\theta=32.294$ \\
\hline 1250 & $\theta=88.031$ & $\theta=46.483$ & $\theta=35.066$ \\
\hline
\end{tabular}

Table 10 and Table 11 shows the effect of fiber density to contact angle. Based on the results, both fiber with orientation $0^{\circ} / 90^{\circ}$ or fiber with orientation $45^{\circ} / 45^{\circ}$ shows that contact angle in the sheet with fiber density of 900 is lower than the sheet with fiber density of 1250 . In addition, wettability can also be observed from the wetting time.

Table 12. Wetting Time for Fiber with Surface Treatment

\begin{tabular}{|c|c|c|}
\hline Fiber Density & Fiber Orientation & Wetting Time (s) \\
\hline \multirow{2}{*}{$0 \% / 90^{\circ}$} & 900 & 19.00 \\
\cline { 2 - 3 } & 1250 & 19.67 \\
\hline \multirow{2}{*}{$45^{\circ} / 45^{\circ}$} & 900 & 36.33 \\
\cline { 2 - 3 } & 1250 & 37.00 \\
\hline
\end{tabular}

Table 12 shows that the sheet with fiber density of 900 has faster wetting time than the sheet with fiber density of 1250 . The sheet with fiber density of 900 has 1 second faster than sheet with fiber density of 1250 . When density of a fiber is tighter, it will more difficult for liquid to enter the surface of fiber because of the small space areas in the surface of fiber. Both of fiber with surface treatment or without surface treatment, it has the same tendency results.

\subsection{The effect of fiber surface treatment}

Surface treatment on fiber with alkali solution $(\mathrm{NaOH}$ $5 \%$ ) decrease the contact angle and wetting time. Fiber with surface treatment has lower contact angle and also faster wetting time than fiber without surface treatment, it is happened in fiber with orientation $0^{\circ} / 90^{\circ}$ and fiber with orientation $45^{\circ} / 45^{\circ}$, and also in sheet with fiber density of 900 and sheet with fiber density of 1250 . The contact angle in surface treated fiber with specification $0^{\circ} / 90^{\circ} / 900$ is 2 degree lower than without surface treatment and 5 degree lower in fiber specification $0 \% / 90^{\circ} / 1250$. Meanwhile, contact angle fiber specification $45^{\circ} / 45^{\circ} / 900$ is 3 degree lower than without surface treatment and 4 degree lower in fiber specification $45^{\circ} / 45^{\circ} / 1250$. The use of alkali solution gives faster wetting time up to 10 second. Surface treatment of fiber with alkali solution results the more rugged fiber with wider space area, which makes it easier for liquid droplet to enter the fiber surface.

\section{Conclusion}

Fiber with orientation $45^{\circ} / 45^{\circ}$ gives lower contact angle but longer wetting time than fiber with orientation $0^{\circ} / 90^{\circ}$. For orientation $45^{\circ} / 45^{\circ}$, the differences wetting time is 20 second longer than orientation $0^{\circ} / 90^{\circ}$. The sheet with fiber density of 900 has lower contact angle and faster wetting time than the sheet with fiber density of 1250 . The wetting time of the sheet with fiber density of 900 is 7 second faster than the sheet with fiber density of 1250 . The surface treatment with $\mathrm{NaOH} 5 \%$ has the faster wetting time for 10 second.

\section{References}

1. K. Adi, S. Firdausi, R. Gernowo, B, Rahardho, I, Siena, A. Putranto. Sistem Pencitraan Mikroskop Digital Untuk Identifikasi Bakteri Tuberkulosis. (2012)

2. Q. Benard, M. Fois, M. Grisel. Roughness and Fibre Reinforcement Effect Onto Wettability of Composite Surfaces. (2006)

3. B. Bhusan. J. of Phil, 1631-1672. (2009)

4. H. Chen, B. Fei, G. Wang, H. Cheng. Contact Angles of Single Fibers Measured in Different Temperature and Related Humidity. (2010)

5. S. F. Chini, A. Amirfazli. Colloids and Surfaces. 177-178. (2011)

6. B. Flinn, A. C. Tracey. Improving Adhesive Bonding of Composite Through Surface Characterization. (2012)

7. M. Gharechahi. J. Biomat. Nanotech. 541-544. (2012)

8. D. Gustri, Ratnawulan, Gusnedi. Pillar. Phys. 09-16. (2014)

9. A.W. Hefer. Adhesion in Bitumen-Aggregate System and Quantification of The Effects of Water on The Adhesive Bond. (2004)

10. M. Hyers. Stress Analysis of Fiber Reinforced Composite Materials. S.1. (1998)

11. Y. Yuan, T. R, Lee. Surf. Sci. Tech. 3-34. (2013) 